TAIWANESE JOURNAL OF MATHEMATICS

Vol. 11, No. 5, pp. 1383-1395, December 2007

This paper is available online at http://www.math.nthu.edu.tw/tjm/

\title{
ON SUPERDERIVATIONS AND LOCAL SUPERDERIVATIONS
}

\author{
Ajda Fošner and Maja Fošner
}

\begin{abstract}
In this paper we describe superderivations in certain superalgebras by their actions on elements satisfying some special conditions. One of the main results is applied to local superderivations on some certain superalgebras.
\end{abstract}

\section{INTRODUCTION}

Throughout the paper, by an algebra we shall mean an algebra over a fixed unital commutative ring $\Phi$, and we shall assume that $\Phi$ contains the element $\frac{1}{2}$ (i.e. $1+1$ is an invertible element).

Let $\mathcal{A}$ be an algebra and let $\mathcal{M}$ be an $\mathcal{A}$-bimodule. Recall that a derivation is a $\Phi$-linear map $d: \mathcal{A} \rightarrow \mathcal{M}$ such that $d(x y)=d(x) y+x d(y)$ for all $x, y \in \mathcal{A}$. In [2] Bresar characterized derivations in certain rings containing noncentral idempotents by their actions on elements satisfying some special conditions. As an application he obtained some new results on local derivations (they are defined below). The main goal of this paper is to prove the superalgebra version of these results. It should be also mentioned that several ideas from [2] will be used in our proofs.

Let $\mathcal{A}$ be an associative superalgebra, that is, a $\mathbb{Z}_{2}$-graded associative algebra. This means that there exist $\Phi$-submodules $\mathcal{A}_{0}$ and $\mathcal{A}_{1}$ of $\mathcal{A}$ such that $\mathcal{A}=\mathcal{A}_{0} \oplus \mathcal{A}_{1}$ and $\mathcal{A}_{0} \mathcal{A}_{0} \subseteq \mathcal{A}_{0}\left(\mathcal{A}_{0}\right.$ is a subalgebra of $\left.\mathcal{A}\right), \mathcal{A}_{0} \mathcal{A}_{1} \subseteq \mathcal{A}_{1}, \mathcal{A}_{1} \mathcal{A}_{0} \subseteq \mathcal{A}_{1}\left(\mathcal{A}_{1}\right.$ is an $\mathcal{A}_{0}$-bimodule), and $\mathcal{A}_{1} \mathcal{A}_{1} \subseteq \mathcal{A}_{0}$. We say that $\mathcal{A}_{0}$ is the even and $\mathcal{A}_{1}$ is the odd part of $\mathcal{A}$. An element $x \in \mathcal{A}_{i}, i=0$ or $i=1$, is said to be homogeneous of degree $i$. In this case we write $|x|=i$. The set of all homogeneous elements of $\mathcal{A}$ will be denoted by $\mathcal{H}(\mathcal{A})$. An ideal $\mathcal{I}$ of $\mathcal{A}$ is said to be graded if $\mathcal{I}=\mathcal{I}_{0} \oplus \mathcal{I}_{1}$, where $\mathcal{I}_{0}=\mathcal{I} \cap \mathcal{A}_{0}$ and $\mathcal{I}_{1}=\mathcal{I} \cap \mathcal{A}_{1}$. A superalgebra $\mathcal{A}$ is called prime if the product of any two nonzero graded ideals in $\mathcal{A}$ is nonzero, and is called simple if its only graded ideals are 0 and $\mathcal{A}$. An $\mathcal{A}$-bimodule $\mathcal{M}$ is an $\mathcal{A}$-superbimodule if $\mathcal{M}=\mathcal{M}_{0} \oplus \mathcal{M}_{1}$ and the multiplication should be self-explanatory.

Received October 4, 2005, accepted January 23, 2006.

Communicated by Wen-Fong Ke.

2000 Mathematics Subject Classification: 17A70, 16W25.

Key words and phrases: Superalgebras, Superderivations, Local superderivations 
A superderivation of degree 0 is a $\Phi$-linear map $d_{0}: \mathcal{A} \rightarrow \mathcal{M}$ such that $d_{0}\left(\mathcal{A}_{0}\right) \subseteq \mathcal{M}_{0}, d_{0}\left(\mathcal{A}_{1}\right) \subseteq \mathcal{M}_{1}$ and $d_{0}(x y)=d_{0}(x) y+x d_{0}(y)$ for all $x, y \in \mathcal{H}(\mathcal{A})$. This is actually a derivation from $\mathcal{A}$ to $\mathcal{M}$. A superderivation of degree 1 is a $\Phi$-linear map $d_{1}: \mathcal{A} \rightarrow \mathcal{M}$ such that $d_{1}\left(\mathcal{A}_{0}\right) \subseteq \mathcal{M}_{1}, d_{1}\left(\mathcal{A}_{1}\right) \subseteq \mathcal{M}_{0}$ and $d_{1}(x y)=$ $d_{1}(x) y+(-1)^{|x|} x d_{1}(y)$ for all $x, y \in \mathcal{H}(\mathcal{A})$. A superderivation $d: \mathcal{A} \rightarrow \mathcal{M}$ is a sum of superderivations $d_{0}$ and $d_{1}$.

Let $\mathcal{A}$ be an algebra and let $\mathcal{M}$ be an $\mathcal{A}$-bimodule. A local derivation is a $\Phi$-linear map $d: \mathcal{A} \rightarrow \mathcal{M}$ such that for every $x \in \mathcal{A}$ there exists a derivation $d_{x}: \mathcal{A} \rightarrow \mathcal{M}$ such that $d(x)=d_{x}(x)$. The standard problem, initiated by Kadison [9] and Larson and Sourour [10], is to find conditions implying that a local derivation is actually a derivation. A number of papers dealing with this problem have been published, see for example [1, 3, 6-8, 11-18].

Motivated by these results we introduce the following definition.

Definition 1.1. Let $\mathcal{A}$ be an associative superalgebra and let $\mathcal{M}$ be an $\mathcal{A}$ superbimodule. Further, let $i=0$ or $i=1$. A $\Phi$-linear map $d_{i}: \mathcal{A} \rightarrow \mathcal{M}$ is called a local superderivation of degree $i$ if for every $x \in \mathcal{A}$ there exists a superderivation of degree $i d_{i_{x}}: \mathcal{A} \rightarrow \mathcal{M}$ such that $d_{i}(x)=d_{i_{x}}(x)$. A local superderivation is a sum of a local superderivation of degree 0 and a local superderivation of degree 1 .

The results on local superderivations will be actually obtained as corollaries to our main results in which we shall study maps satisfying certain more general conditions. In order to present these conditions we introduce and fix some notation. Throughout the paper $\mathcal{A}=\mathcal{A}_{0} \oplus \mathcal{A}_{1}$ will be an associative superalgebra and $\mathcal{M}=$ $\mathcal{M}_{0} \oplus \mathcal{M}_{1}$ will be an $\mathcal{A}$-superbimodule. By $\mathcal{E}$ we denote the set $\mathcal{E}=\mathcal{E}_{0} \oplus \mathcal{E}_{1}$, where $\mathcal{E}_{0}=\left\{e \in \mathcal{A}_{0} \mid e^{2}=e\right\}\left(\mathcal{E}_{0}\right.$ is the set of all idempotents in $\left.\mathcal{A}_{0}\right)$ and $\mathcal{E}_{1}=\left\{e \in \mathcal{A}_{1} \mid\right.$ there exists $e^{\prime} \in \mathcal{E}_{0}$ such that $\left.\left(e^{\prime}+e\right)^{2}=e^{\prime}+e\right\}$. Note that the condition $\left(e^{\prime}+e\right)^{2}=e^{\prime}+e, e^{\prime} \in \mathcal{E}_{0}, e \in \mathcal{E}_{1}$, implies $e^{2}=0$ and $e^{\prime} e+e e^{\prime}=e$. Further, by $\mathcal{R}=\mathcal{R}_{0} \oplus \mathcal{R}_{1}$ we denote the subsuperalgebra of $\mathcal{A}$ generated by $\mathcal{E}$, and by $\mathcal{I}=\mathcal{I}_{0} \oplus \mathcal{I}_{1}$ we denote the graded ideal generated by $\left[\mathcal{E}_{0}, \mathcal{A}\right]$. Here, [ . , . ] denotes the commutator.

The next simple lemma will show us that the existence of merely one noncentral idempotent in $\mathcal{A}_{0}$ implies that $\mathcal{R}$ contains a nonzero graded ideal. By a central idempotent we mean an idempotent $e$ such that $[e, x]=0$ for all $x \in \mathcal{A}$.

Lemma 1.2. $\mathcal{I} \subseteq \mathcal{R}$.

Proof. Let $e \in \mathcal{E}_{0}$ and $x \in \mathcal{H}(\mathcal{A})$. Note that the elements $e+e x-e x e$ and $e+x e-e x e$ are also in $\mathcal{E}$. Namely, if $x \in \mathcal{A}_{0}$ then clearly $e+e x-e x e, e+x e-e x e \in$ $\mathcal{E}_{0}$. In the case when $x \in \mathcal{A}_{1}$ we have $e x-e x e, x e-e x e \in \mathcal{E}_{1}\left(e^{\prime}=e\right)$. Since $\mathcal{E} \subseteq \mathcal{R}$ we get $(e+e x-e x e)-(e+x e-e x e)=[e, x] \in \mathcal{R}$. Using the proof of [2, Lemma 2.1] the result follows. 
We will get particularly nice results for superalgebras $\mathcal{A}$ such that $\mathcal{A}=\mathcal{R}$. So, the natural question that appears is when $\mathcal{A}=\mathcal{R}$. Lemma 1.2 makes it possible for us to give a few examples of such superalgebras:

(a) $\mathcal{A}$ is a simple superalgebra containing a nontrivial idempotent in $\mathcal{A}_{0}$,

(b) $\mathcal{A}$ is a unital superalgebra containing an idempotent $e_{0} \in \mathcal{A}_{0}$ such that graded ideals generated by $e_{0}$ and $1-e_{0}$, respectively, are both equal to $\mathcal{A}$,

(c) $\mathcal{A}=M_{n}(\mathcal{B})$, the superalgebra of all $n \times n$ matrices over any unital algebra $\mathcal{B}$, where $n \geq 2$.

By a nontrivial idempotent we mean an idempotent different from 0 and 1 . Simple superalgebras (as well as prime superalgebras) do not contain nontrivial central idempotents in the even part. Therefore, if $\mathcal{A}$ is a superalgebra of the type (a), we have $\mathcal{I} \neq 0$, which yields $\mathcal{R}=\mathcal{A}$ by Lemma 1.2.

Now assume that $\mathcal{A}$ satisfies the condition (b). Then in particular we have $\sum_{j} x_{j}\left(1-e_{0}\right) y_{j}=e_{0}$ for some $x_{j}, y_{j} \in \mathcal{H}(\mathcal{A})$, which in turn implies $e_{0}=$ $\sum_{j}\left[e_{0}, x_{j}\right]\left(1-e_{0}\right) y_{j} \in \mathcal{I}$. Analogously, $\sum_{j} x_{j}^{\prime} e_{0} y_{j}^{\prime}=1-e_{0}$ for some $x_{j}^{\prime}, y_{j}^{\prime} \in$ $\mathcal{H}(\mathcal{A})$ and hence $1-e_{0}=\sum_{j}\left[x_{j}^{\prime}, e_{0}\right] e_{0} y_{j}^{\prime} \in \mathcal{I}$. Consequently, $1 \in \mathcal{I}$, which implies that $\mathcal{I}=\mathcal{R}=\mathcal{A}$.

Finally, let $\mathcal{A}$ be of the type (c). Then it is not difficult to see that $\mathcal{A}$ satisfies (b). Namely, one can choose, for example, the matrix unit $E_{11}$ for $e_{0}$.

Let $i=0$ or $i=1$ and let $d_{i}: \mathcal{A} \rightarrow \mathcal{M}$ be a $\Phi$-linear map. We shall consider the following conditions:

$$
\begin{array}{ll}
\left(\mathbf{d}_{\mathbf{i}} \mathbf{1}\right) \quad x y=y z=0 \Rightarrow\left(x_{0}+(-1)^{i} x_{1}\right) d_{i}(y) z=0 \\
\\
\left(x=x_{0}+x_{1}, y, z \in \mathcal{A}=\mathcal{A}_{0} \oplus \mathcal{A}_{1}\right), \\
\left(\mathbf{d}_{\mathbf{i}} \mathbf{2}\right) \quad x y=0 \Rightarrow d_{i}(x) y+\left(x_{0}+(-1)^{i} x_{1}\right) d_{i}(y)=0 \\
\\
\left(x=x_{0}+x_{1}, y \in \mathcal{A}=\mathcal{A}_{0} \oplus \mathcal{A}_{1}\right) .
\end{array}
$$

Note that maps satisfying the conditions $\left(d_{0} 2\right)$ and $\left(d_{1} 2\right)$ are clearly special cases of maps satisfying the conditions $\left(d_{0} 1\right)$ and $\left(d_{1} 1\right)$, respectively.

Suppose that $d_{i}: \mathcal{A} \rightarrow \mathcal{M}, i=0,1$, is a superderivation of degree $i$. Pick any $x=x_{0}+x_{1}, y \in \mathcal{A}=\mathcal{A}_{0} \oplus \mathcal{A}_{1}$ such that $x y=0$. It follows that

$$
0=d_{i}(x y)=d_{i}(x) y+\left(x_{0}+(-1)^{i} x_{1}\right) d_{i}(y) .
$$

Further, if $x y=y z=0, z \in \mathcal{A}$, we arrive at

$$
0=d_{i}(x y) z=d_{i}(x) y z+\left(x_{0}+(-1)^{i} x_{1}\right) d_{i}(y) z=\left(x_{0}+(-1)^{i} x_{1}\right) d_{i}(y) z .
$$

Thus, every superderivation of degree 0 satisfies the conditions $\left(d_{0} 1\right)$ and $\left(d_{0} 2\right)$. Similarly, every superderivation of degree 1 satisfies the conditions $\left(d_{1} 1\right)$ and $\left(d_{1} 2\right)$. 
The obvious problem, which is the main issue of this paper, is to show that in appropriate settings the converses are true.

There is a simple link between local superderivations of degree $i, i=0,1$, and these conditions. Namely, if $d_{i}: \mathcal{A} \rightarrow \mathcal{M}$ is a local superderivation of degree $i$, then for all $x=x_{0}+x_{1}, y, z \in \mathcal{A}=\mathcal{A}_{0} \oplus \mathcal{A}_{1}$ we have

$$
\left(x_{0}+(-1)^{i} x_{1}\right) d_{i}(y) z=\left(x_{0}+(-1)^{i} x_{1}\right) d_{i_{y}}(y) z=d_{i_{y}}(x y) z-d_{i_{y}}(x) y z
$$

and hence $\left(x_{0}+(-1)^{i} x_{1}\right) d_{i}(y) z=0$ in the case when $x y=y z=0$. Thus, local superderivations of degree $i$ satisfy $\left(d_{i} 1\right)$.

\section{Conditions $\left(d_{0} 1\right)$ AND $\left(d_{1} 1\right)$}

First we shall consider the conditions $\left(d_{0} 1\right)$ and $\left(d_{1} 1\right)$. The following theorem plays an important role in this section. As we shall see, the results on local superderivations will follow from those on the conditions $\left(d_{0} 1\right)$ and $\left(d_{1} 1\right)$.

Theorem 2.1. Let $\mathcal{A}$ be a unital superalgebra, let $\mathcal{M}$ be a unital $\mathcal{A}$-superbimodule, and let $d_{i}: \mathcal{A} \rightarrow \mathcal{M}, i=0,1$, be a $\Phi$-linear map satisfying $d_{i}\left(\mathcal{A}_{0}\right) \subseteq \mathcal{M}_{i}$, $d_{i}\left(\mathcal{A}_{1}\right) \subseteq \mathcal{M}_{1+i}$ (index modulo 2$),\left(d_{i} 1\right)$, and $d_{i}(1)=0$. Then the restriction of $d_{i}$ to $\mathcal{R}$ is a superderivation of degree $i$. Moreover,

$$
\begin{aligned}
& d_{i}(r x s)+(-1)^{i|r|} r d_{i}(x) s=d_{i}(r x) s+(-1)^{i|r|} r d_{i}(x s) \\
& (r, s \in \mathcal{H}(\mathcal{R}), x \in \mathcal{H}(\mathcal{A})) \\
& \mathcal{I}\left(d_{i}(x y)-d_{i}(x) y-(-1)^{i|x|} x d_{i}(y)\right) \mathcal{I}=0 \\
& (x, y \in \mathcal{H}(\mathcal{A})) .
\end{aligned}
$$

Proof. Let $e$ and $f$ be idempotents in $\mathcal{A}$ and pick any $x \in \mathcal{A}$. It is easy to see that the following identities hold true

$$
\begin{aligned}
& (1-e) \cdot e x f=e x f \cdot(1-f)=0, \\
& e \cdot(1-e) x f=(1-e) x f \cdot(1-f)=0, \\
& (1-e) \cdot e x(1-f)=e x(1-f) \cdot f=0, \\
& e \cdot(1-e) x(1-f)=(1-e) x(1-f) \cdot f=0 .
\end{aligned}
$$

Setting $e=e_{0}, f=f_{0} \in \mathcal{E}_{0}$ in (3) and using $\left(d_{i} 1\right)$ we obtain

$$
\begin{aligned}
& \left(1-e_{0}\right) d_{i}\left(e_{0} x f_{0}\right)\left(1-f_{0}\right)=0, \\
& e_{0} d_{i}\left(\left(1-e_{0}\right) x f_{0}\right)\left(1-f_{0}\right)=0, \\
& \left(1-e_{0}\right) d_{i}\left(e_{0} x\left(1-f_{0}\right)\right) f_{0}=0, \\
& e_{0} d_{i}\left(\left(1-e_{0}\right) x\left(1-f_{0}\right)\right) f_{0}=0 .
\end{aligned}
$$


It follows that

$$
d_{i}\left(e_{0} x f_{0}\right)+e_{0} d_{i}(x) f_{0}=d_{i}\left(e_{0} x\right) f_{0}+e_{0} d_{i}\left(x f_{0}\right)
$$

for all $e_{0}, f_{0} \in \mathcal{E}_{0}$ and $x \in \mathcal{A}$.

Further, let $e=e_{0} \in \mathcal{E}_{0}$ and let $f=f_{0}+f_{1} \in \mathcal{A}$ be an idempotent with $f_{0} \in \mathcal{E}_{0}$ and $f_{1} \in \mathcal{E}_{1}$. Using (3) and $\left(d_{i} 1\right)$ we arrive at

$$
\begin{aligned}
& \left(1-e_{0}\right) d_{i}\left(e_{0} x f\right)(1-f)=0, \\
& e_{0} d_{i}\left(\left(1-e_{0}\right) x f\right)(1-f)=0, \\
& \left(1-e_{0}\right) d_{i}\left(e_{0} x(1-f)\right) f=0, \\
& e_{0} d_{i}\left(\left(1-e_{0}\right) x(1-f)\right) f=0 .
\end{aligned}
$$

Hence $d_{i}\left(e_{0} x f\right)+e_{0} d_{i}(x) f=d_{i}\left(e_{0} x\right) f+e_{0} d_{i}(x f)$. From (4) we get

$$
d_{i}\left(e_{0} x f_{1}\right)+e_{0} d_{i}(x) f_{1}=d_{i}\left(e_{0} x\right) f_{1}+e_{0} d_{i}\left(x f_{1}\right)
$$

for all $e_{0} \in \mathcal{E}_{0}, f_{1} \in \mathcal{E}_{1}$ and $x \in \mathcal{A}$.

Now pick $f=f_{0} \in \mathcal{E}_{0}$ and an idempotent $e=e_{0}+e_{1} \in \mathcal{A}$, where $e_{0} \in \mathcal{E}_{0}$ and $e_{1} \in \mathcal{E}_{1}$. Again using (3) and $\left(d_{i} 1\right)$ we obtain

$$
\begin{aligned}
& \left(1-e_{0}-(-1)^{i} e_{1}\right) d_{i}\left(e x f_{0}\right)\left(1-f_{0}\right)=0, \\
& \left(e_{0}+(-1)^{i} e_{1}\right) d_{i}\left((1-e) x f_{0}\right)\left(1-f_{0}\right)=0, \\
& \left(1-e_{0}-(-1)^{i} e_{1}\right) d_{i}\left(e x\left(1-f_{0}\right)\right) f_{0}=0, \\
& \left(e_{0}+(-1)^{i} e_{1}\right) d_{i}\left((1-e) x\left(1-f_{0}\right)\right) f_{0}=0 .
\end{aligned}
$$

By (3) we infer

$$
d_{i}\left(e_{1} x f_{0}\right)+(-1)^{i} e_{1} d_{i}(x) f_{0}=d_{i}\left(e_{1} x\right) f_{0}+(-1)^{i} e_{1} d_{i}\left(x f_{0}\right)
$$

for all $e_{1} \in \mathcal{E}_{1}, f_{0} \in \mathcal{E}_{0}$ and $x \in \mathcal{A}$.

Finally, let $e=e_{0}+e_{1} \in \mathcal{A}$ and $f=f_{0}+f_{1} \in \mathcal{A}$ be idempotents with $e_{0}, f_{0} \in \mathcal{E}_{0}$ and $e_{1}, f_{1} \in \mathcal{E}_{1}$. Using the same procedure as above we arrive at

$$
d_{i}\left(e_{1} x f_{1}\right)+(-1)^{i} e_{1} d_{i}(x) f_{1}=d_{i}\left(e_{1} x\right) f_{1}+(-1)^{i} e_{1} d_{i}\left(x f_{1}\right)
$$

for all $e_{1}, f_{1} \in \mathcal{E}_{1}$ and $x \in \mathcal{A}$. By (4), (5), (6), and (7) we proved that

$$
d_{i}(e x f)+(-1)^{i|e|} e d_{i}(x) f=d_{i}(e x) f+(-1)^{i|e|} e d_{i}(x f)
$$

for all $e, f \in \mathcal{H}(\mathcal{E})$ and $x \in \mathcal{H}(\mathcal{A})$. In particular, by setting $x=1$ and using $d_{i}(1)=0$ we get

$$
d_{i}(e f)=d_{i}(e) f+(-1)^{i|e|} e d_{i}(f)
$$


for all $e, f \in \mathcal{H}(\mathcal{E})$.

In the next step we will prove that the restriction of $d_{i}$ to $\mathcal{R}$ is a superderivation of degree $i$. All we have to do is to show that

$$
\begin{aligned}
& d_{i}\left(e_{1} e_{2} \ldots e_{n}\right)= \\
& d_{i}\left(e_{1}\right) e_{2} \ldots e_{n-1} e_{n}+(-1)^{i\left|e_{1}\right|} e_{1} d_{i}\left(e_{2}\right) e_{3} \ldots e_{n-1} e_{n}+ \\
& +\ldots+(-1)^{i\left|e_{1} e_{2} \ldots e_{n-1}\right|} e_{1} e_{2} \ldots e_{n-1} d_{i}\left(e_{n}\right)
\end{aligned}
$$

holds true for all $n \in \mathbb{N} \backslash\{1\}$, where $e_{1}, e_{2}, \ldots, e_{n} \in \mathcal{H}(\mathcal{E})$. We shall use the induction on $n$. By (9) our assertion is already proved for $n=2$. Let $2<n$ and assume that the statement holds true for all positive integers smaller than $n$. Using (8) we have

$$
\begin{aligned}
& d_{i}\left(e_{1} e_{2} \ldots e_{n}\right)=d_{i}\left(e_{1} e_{2} \ldots e_{n-1}\right) e_{n}+ \\
& +(-1)^{i\left|e_{1}\right|} e_{1} d_{i}\left(e_{2} \ldots e_{n}\right)-(-1)^{i\left|e_{1}\right|} e_{1} d_{i}\left(e_{2} \ldots e_{n-1}\right) e_{n}
\end{aligned}
$$

for all $e_{1}, e_{2}, \ldots, e_{n} \in \mathcal{H}(\mathcal{E})$. This together with induction hypothesis yields (10). Thus we proved that the restriction of $d_{i}$ to $\mathcal{R}$ is a superderivation of degree $i$, as desired.

For any $a \in \mathcal{H}(\mathcal{A})$ we shall denote the sets

$$
\begin{aligned}
\mathcal{S}_{a}= & \left\{r \in \mathcal{H}(\mathcal{R}) \mid d_{i}(r x a)+(-1)^{i|r|} r d_{i}(x) a=d_{i}(r x) a+(-1)^{i|r|} r d_{i}(x a)\right. \\
& \text { for all } x \in \mathcal{H}(\mathcal{A})\}, \\
\mathcal{T}_{a}= & \left\{s \in \mathcal{H}(\mathcal{R}) \mid d_{i}(a x s)+(-1)^{i|a|} a d_{i}(x) s=d_{i}(a x) s+(-1)^{i|a|} a d_{i}(x s)\right. \\
& \text { for all } x \in \mathcal{H}(\mathcal{A})\} .
\end{aligned}
$$

Given $r, r^{\prime} \in \mathcal{S}_{a}$ we have

$$
\begin{aligned}
& d_{i}\left(r r^{\prime} x a\right)=d_{i}\left(r r^{\prime} x\right) a+(-1)^{i|r|} r d_{i}\left(r^{\prime} x a\right)-(-1)^{i|r|} r d_{i}\left(r^{\prime} x\right) a \\
& =d_{i}\left(r r^{\prime} x\right) a+(-1)^{i|r|} r\left(d_{i}\left(r^{\prime} x\right) a+(-1)^{i\left|r^{\prime}\right|} r^{\prime} d_{i}(x a)-(-1)^{i\left|r^{\prime}\right|} r^{\prime} d_{i}(x) a\right) \\
& -(-1)^{i|r|} r d_{i}\left(r^{\prime} x\right) a=d_{i}\left(r r^{\prime} x\right) a+(-1)^{i\left|r r^{\prime}\right|} r r^{\prime} d_{i}(x a)-(-1)^{i\left|r r^{\prime}\right|} r r^{\prime} d_{i}(x) a
\end{aligned}
$$

for all $x \in \mathcal{H}(\mathcal{A})$. Thus we showed that $r r^{\prime} \in \mathcal{S}_{a}$ for every pair $r, r^{\prime} \in \mathcal{S}_{a}$. Similarly, we can show that $s s^{\prime} \in \mathcal{T}_{a}$ for every pair $s, s^{\prime} \in \mathcal{T}_{a}$. By (8) we have $\mathcal{H}(\mathcal{E}) \subseteq \mathcal{S}_{f}$ for all $f \in \mathcal{H}(\mathcal{E})$, and consequently $\mathcal{H}(\mathcal{R}) \subseteq \mathcal{S}_{f}$ for all $f \in \mathcal{H}(\mathcal{E})$. Therefore, $f \in \mathcal{T}_{r}$ for every $f \in \mathcal{H}(\mathcal{E})$ and every $r \in \mathcal{H}(\mathcal{R})$. This yields that $\mathcal{H}(\mathcal{R}) \subseteq \mathcal{T}_{r}$ for every $r \in \mathcal{H}(\mathcal{R})$. Thus we proved (1).

Now pick $u \in \mathcal{H}(\mathcal{I}), x \in \mathcal{H}(\mathcal{A})$, and $s \in \mathcal{H}(\mathcal{R})$. Then $u x \in \mathcal{H}(\mathcal{I}) \subseteq \mathcal{H}(\mathcal{R})$ by Lemma 1.2. Since $d_{i}$ is a superderivation of degree $i$ on $\mathcal{R}$, we have

$$
d_{i}(u x s)=d_{i}(u x) s+(-1)^{i|u x|} u x d_{i}(s) .
$$


On the other hand, since $u, s \in \mathcal{H}(\mathcal{R})$, it follows from (1) that

$$
d_{i}(u x s)=d_{i}(u x) s+(-1)^{i|u|} u d_{i}(x s)-(-1)^{i|u|} u d_{i}(x) s .
$$

Comparing the last two identities we obtain

$$
u\left(d_{i}(x s)-d_{i}(x) s-(-1)^{i|x|} x d_{i}(s)\right)=0
$$

for all $u \in \mathcal{H}(\mathcal{I}), x \in \mathcal{H}(\mathcal{A})$, and $s \in \mathcal{H}(\mathcal{R})$. Now let $y \in \mathcal{H}(\mathcal{A})$ and $v \in \mathcal{H}(\mathcal{I})$. Since $v \in \mathcal{H}(\mathcal{R})$ we get from (11)

$$
u\left(d_{i}((x y) v)-d_{i}(x y) v-(-1)^{i|x y|} x y d_{i}(v)\right)=0 .
$$

On the other hand, since $y v, v \in \mathcal{I} \subseteq \mathcal{R}$ and $u x \in \mathcal{I}$, (11) implies

$$
\begin{aligned}
0 & =u\left(d_{i}(x(y v))-d_{i}(x) y v-(-1)^{i|x|} x d_{i}(y v)\right) \\
& =u\left(d_{i}(x(y v))-d_{i}(x) y v-(-1)^{i|x|} x\left(d_{i}(y) v+(-1)^{i|y|} y d_{i}(v)\right)\right) .
\end{aligned}
$$

Comparing the last two identities we get (2). Thereby the proof is completed.

Corollary 2.2. If $\mathcal{R}=\mathcal{A}$, then $d_{i}: \mathcal{A} \rightarrow \mathcal{M}, i=0,1$, is a superderivation of degree $i$.

Corollary 2.3. Let $d_{i}: \mathcal{A} \rightarrow \mathcal{A}, i=0,1$, be a $\Phi$-linear map satisfying $d_{i}\left(\mathcal{A}_{0}\right) \subseteq \mathcal{A}_{i}, d_{i}\left(\mathcal{A}_{1}\right) \subseteq \mathcal{A}_{1+i}$ (index modulo 2 ), $\left(d_{i} 1\right)$, and $d_{i}(1)=0$. If $\mathcal{A}$ is a prime superalgebra containing a nontrivial idempotent in $\mathcal{A}_{0}$, then $d_{i}$ is a superderivation of degree $i$.

Note that the conclusions of Theorem 2.1 hold also for local superderivations $d_{i}: \mathcal{A} \rightarrow \mathcal{M}, i=0,1$, of degree $i$.

Now let $\mathcal{A}$ be a nonunital superalgebra and let $d_{i}: \mathcal{A} \rightarrow \mathcal{M}, i=0,1$, be a local superderivation of degree $i$. Then we can consider a superalgebra $\mathcal{A}^{\prime}$ obtained by adjoining a unity in $\mathcal{A}$. Setting $1 m=m=m 1$ for every $m \in \mathcal{M}, \mathcal{M}$ then becomes a unital $\mathcal{A}^{\prime}$-superbimodule. Extend $d_{i}$ to $\mathcal{A}^{\prime}$ by defining $d_{i}(1)=0$. Note that $d_{i}$ is a local superderivation of degree $i$ on $\mathcal{A}^{\prime}$. Therefore the conclusions of Theorem 2.1 hold for local superderivations $d_{i}: \mathcal{A} \rightarrow \mathcal{M}$ even when $\mathcal{A}$ and $\mathcal{M}$ are not unital. In particular, every superalgebra $\mathcal{A}$ with a noncentral idempotent in $\mathcal{A}_{0}$ contains a nonzero graded ideal $\mathcal{I}$ such that every local superderivation of degree $i d_{i}: \mathcal{A} \rightarrow \mathcal{M}, i=0,1$, is a superderivation of degree $i$ on $\mathcal{I}$. Thus, every local superderivation from $\mathcal{A}$ to $\mathcal{M}$ is a superderivation on $\mathcal{I}$.

Corollary 2.4. If $\mathcal{R}=\mathcal{A}$, then every local superderivation $d: \mathcal{A} \rightarrow \mathcal{M}$ is a superderivation. 
Proof. Let $d_{i}: \mathcal{A} \rightarrow \mathcal{M}, i=0,1$, be a local superderivation of degree $i$. Hence, $d_{i}$ satisfies $\left(d_{i} 1\right)$. According to Theorem 2.1 it follows that $d_{i}$ is a superderivation of degree $i$ on $\mathcal{A}$. Consequently, every local superderivation $d: \mathcal{A} \rightarrow \mathcal{M}$ is a superderivation.

Corollary 2.5. Let $\mathcal{A}$ be a prime superalgebra containing a nontrivial idempotent in $\mathcal{A}_{0}$. Then every local superderivation $d: \mathcal{A} \rightarrow \mathcal{A}$ is a superderivation.

Proof. According to our assumption $\mathcal{A}$ contains a nonzero graded ideal $\mathcal{I}$. Now let $d_{i}: \mathcal{A} \rightarrow \mathcal{A}, i=0,1$, be a local superderivation of degree $i$. Using Theorem 2.1 it follows that (2) holds true. Since $\mathcal{A}$ is prime, $d_{i}$ is a superderivation of degree $i$ on $\mathcal{A}$. Consequently, every local superderivation $d: \mathcal{A} \rightarrow \mathcal{A}$ is a superderivation.

\section{Conditions $\left(d_{0} 2\right)$ AND $\left(d_{1} 2\right)$}

In this section we shall consider the conditions $\left(d_{0} 2\right)$ and $\left(d_{1} 2\right)$. Clearly, the condition $\left(d_{0} 2\right)$ implies the condition $\left(d_{0} 1\right)$ and $\left(d_{1} 2\right)$ implies $\left(d_{1} 1\right)$. Thus, the results of the previous section give some conclusions for maps satisfying $\left(d_{0} 2\right)$ or $\left(d_{1} 2\right)$. However, as we shall see, we will obtain some stronger results. In particular, we will not assume that our superalgebras and superbimodules are unital.

Theorem 3.1. Let $\mathcal{A}$ be a superalgebra, let $\mathcal{M}$ be an $\mathcal{A}$-superbimodule, and let $d_{i}: \mathcal{A} \rightarrow \mathcal{M}, i=0,1$, be a $\Phi$-linear map satisfying $d_{i}\left(\mathcal{A}_{0}\right) \subseteq \mathcal{M}_{i}, d_{i}\left(\mathcal{A}_{1}\right) \subseteq \mathcal{M}_{1+i}$ (index modulo 2$)$, and $\left(d_{i} 2\right)$. Then

$$
\begin{aligned}
& d_{i}(x r) z+(-1)^{i|x r|} x r d_{i}(z)=d_{i}(x) r z+(-1)^{i|x|} x d_{i}(r z) \\
& (x, z \in \mathcal{H}(\mathcal{A}), r \in \mathcal{H}(\mathcal{R})), \\
& (-1)^{i|w t z|} w t z\left(d_{i}(x y)-d_{i}(x) y-(-1)^{i|x|} x d_{i}(y)\right) \\
& =\left(d_{i}(w t)-d_{i}(w) t-(-1)^{i|w|} w d_{i}(t)\right) z x y \\
& \left(t \in \mathcal{H}\left(\mathcal{A}^{2} \mathcal{I}\right), x, y, z, w \in \mathcal{H}(\mathcal{A})\right) .
\end{aligned}
$$

Proof. Let $e$ be an idempotent in $\mathcal{A}$ and let $x, z \in \mathcal{H}(\mathcal{A})$. Then

$$
\begin{aligned}
& (x-x e) \cdot e z=0, \\
& x e \cdot(z-e z)=0 .
\end{aligned}
$$

First suppose that $e=e_{0} \in \mathcal{E}_{0}$. Using $\left(d_{i} 2\right)$ it follows that

$$
\begin{aligned}
& d_{i}\left(x-x e_{0}\right) e_{0} z+(-1)^{i\left|x e_{0}\right|}\left(x-x e_{0}\right) d_{i}\left(e_{0} z\right)=0, \\
& d_{i}\left(x e_{0}\right)\left(z-e_{0} z\right)+(-1)^{i\left|x e_{0}\right|} x e_{0} d_{i}\left(z-e_{0} z\right)=0 .
\end{aligned}
$$


These two identities imply

$$
d_{i}\left(x e_{0}\right) z+(-1)^{i\left|x e_{0}\right|} x e_{0} d_{i}(z)=d_{i}(x) e_{0} z+(-1)^{i|x|} x d_{i}\left(e_{0} z\right)
$$

for all $x, z \in \mathcal{H}(\mathcal{A})$ and $e_{0} \in \mathcal{E}_{0}$. Now let $e=e_{0}+e_{1} \in \mathcal{A}$ be an idempotent, where $e_{0} \in \mathcal{E}_{0}$ and $e_{1} \in \mathcal{E}_{1}$. Using (14), $\left(d_{i} 2\right)$, and (15) it follows that

$$
d_{i}\left(x e_{1}\right) z+(-1)^{i\left|x e_{1}\right|} x e_{1} d_{i}(z)=d_{i}(x) e_{1} z+(-1)^{i|x|} x d_{i}\left(e_{1} z\right)
$$

for all $x, z \in \mathcal{H}(\mathcal{A})$ and $e_{1} \in \mathcal{E}_{1}$. Thus we proved

$$
d_{i}(x e) z+(-1)^{i|x e|} x e d_{i}(z)=d_{i}(x) e z+(-1)^{i|x|} x d_{i}(e z)
$$

for all $x, z \in \mathcal{H}(\mathcal{A})$ and $e \in \mathcal{H}(\mathcal{E})$. Let

$$
\begin{aligned}
\mathcal{T}= & \left\{r \in \mathcal{H}(\mathcal{A}) \mid d_{i}(x r) z+(-1)^{i|x r|} x r d_{i}(z)=d_{i}(x) r z+(-1)^{i|x|} x d_{i}(r z)\right. \\
& \quad \text { for all } x, z \in \mathcal{H}(\mathcal{A})\} .
\end{aligned}
$$

Given $r, r^{\prime} \in \mathcal{T}$ and $x, z \in \mathcal{H}(\mathcal{A})$ we have

$$
\begin{aligned}
d_{i}\left(x r r^{\prime}\right) z & =d_{i}(x r) r^{\prime} z+(-1)^{i|x r|} x r d_{i}\left(r^{\prime} z\right)-(-1)^{i\left|x r r^{\prime}\right|} x r r^{\prime} d_{i}(z) \\
& =d_{i}(x) r r^{\prime} z+(-1)^{i|x|} x d_{i}\left(r r^{\prime} z\right)-(-1)^{i\left|x r r^{\prime}\right|} x r r^{\prime} d_{i}(z) .
\end{aligned}
$$

Therefore we proved that $r r^{\prime} \in \mathcal{T}$. Since $\mathcal{H}(\mathcal{E}) \subseteq \mathcal{T}$ by (16), we get $\mathcal{H}(\mathcal{R}) \subseteq \mathcal{T}$. This proves (12).

Let $u \in \mathcal{H}(\mathcal{I})$ and $x, y, z, w, w^{\prime}, w^{\prime \prime} \in \mathcal{H}(\mathcal{A})$. Then $u z x, w^{\prime \prime} u z, w^{\prime} w^{\prime \prime} u \in$ $\mathcal{H}(\mathcal{I}) \subseteq \mathcal{H}(\mathcal{R})$ by Lemma 1.2. Using (12) we obtain

$$
\begin{aligned}
w w^{\prime} w^{\prime \prime} d_{i}(u z x y)= & (-1)^{i\left|w^{\prime \prime}\right|} w w^{\prime} d_{i}\left(w^{\prime \prime} u z x\right) y+(-1)^{i|u z x|} w w^{\prime} w^{\prime \prime} u z x d_{i}(y) \\
& -(-1)^{i\left|w^{\prime \prime}\right|} w w^{\prime} d_{i}\left(w^{\prime \prime}\right) u z x y \\
= & (-1)^{i\left|w^{\prime \prime}\right|} w\left((-1)^{i\left|w^{\prime}\right|} d_{i}\left(w^{\prime} w^{\prime \prime} u z\right) x+(-1)^{i\left|w^{\prime \prime} u z\right|} w^{\prime} w^{\prime \prime} u z d_{i}(x)\right. \\
& \left.-(-1)^{i\left|w^{\prime}\right|} d_{i}\left(w^{\prime}\right) w^{\prime \prime} u z x\right) y+(-1)^{i|u z x|} w w^{\prime} w^{\prime \prime} u z x d_{i}(y) \\
& -(-1)^{i\left|w^{\prime \prime}\right|} w w^{\prime} d_{i}\left(w^{\prime \prime}\right) u z x y \\
= & (-1)^{i\left|w^{\prime \prime} w^{\prime}\right|}\left((-1)^{i|w|} d_{i}\left(w w^{\prime} w^{\prime \prime} u\right) z+(-1)^{i\left|w^{\prime} w^{\prime \prime} u\right|} w w^{\prime} w^{\prime \prime} u d_{i}(z)\right. \\
& \left.-(-1)^{i|w|} d_{i}(w) w^{\prime} w^{\prime \prime} u z\right) x y+(-1)^{i|u z|} w w^{\prime} w^{\prime \prime} u z d_{i}(x) y \\
& -(-1)^{i\left|w^{\prime \prime} w^{\prime}\right|} w d_{i}\left(w^{\prime}\right) w^{\prime \prime} u z x y+(-1)^{i|u z x|} w w^{\prime} w^{\prime \prime} u z x d_{i}(y) \\
& -(-1)^{i\left|w^{\prime \prime}\right|} w w^{\prime} d_{i}\left(w^{\prime \prime}\right) u z x y .
\end{aligned}
$$

On the other hand, since $u z, w^{\prime \prime} u \in \mathcal{H}(\mathcal{R})$, we arrive at 


$$
\begin{aligned}
w w^{\prime} w^{\prime \prime} d_{i}(u z x y)= & (-1)^{i\left|w^{\prime \prime}\right|} w w^{\prime} d_{i}\left(w^{\prime \prime} u z\right) x y+(-1)^{i|u z|} w w^{\prime} w^{\prime \prime} u z d_{i}(x y) \\
& -(-1)^{i\left|w^{\prime \prime}\right|} w w^{\prime} d_{i}\left(w^{\prime \prime}\right) u z x y \\
= & (-1)^{i\left|w^{\prime \prime}\right|} w\left((-1)^{i\left|w^{\prime}\right|} d_{i}\left(w^{\prime} w^{\prime \prime} u\right) z\right. \\
& \left.+(-1)^{i\left|w^{\prime \prime} u\right|} w^{\prime} w^{\prime \prime} u d_{i}(z)-(-1)^{i\left|w^{\prime}\right|} d_{i}\left(w^{\prime}\right) w^{\prime \prime} u z\right) x y \\
& +(-1)^{i|u z|} w w^{\prime} w^{\prime \prime} u z d_{i}(x y)-(-1)^{i\left|w^{\prime \prime}\right|} w w^{\prime} d_{i}\left(w^{\prime \prime}\right) u z x y .
\end{aligned}
$$

Comparing so obtained identities and write $t=w^{\prime} w^{\prime \prime} u$ we get

$$
\begin{aligned}
& (-1)^{i|w t z|} w t z\left(d_{i}(x y)-d_{i}(x) y-(-1)^{i|x|} x d_{i}(y)\right) \\
= & \left(d_{i}(w t)-d_{i}(w) t-(-1)^{i|w|} w d_{i}(t)\right) z x y
\end{aligned}
$$

for all $t \in \mathcal{H}\left(\mathcal{A}^{2} \mathcal{I}\right), x, y, z, w \in \mathcal{H}(\mathcal{A})$. Thereby the proof is completed.

In the case when $\mathcal{A}=\mathcal{R},(12)$ can be read as

$$
\begin{aligned}
& d_{i}(x y) z+(-1)^{i|x y|} x y d_{i}(z)=d_{i}(x) y z+(-1)^{i|x|} x d_{i}(y z) \\
& (x, y, z \in \mathcal{H}(\mathcal{A})) .
\end{aligned}
$$

Assume that $\mathcal{A}$ and $\mathcal{M}$ are unital. Let $x=z=1$. Using (17) we obtain $\lambda=$ $d_{i}(1) \in \mathcal{Z}(\mathcal{M})=\left\{m \in \mathcal{H}(\mathcal{M}) \mid(-1)^{i|x|} x m=m x\right.$ for all $\left.x \in \mathcal{H}(\mathcal{A})\right\}$. If $i=0$, then $\mathcal{Z}(\mathcal{M})$ is actually the center of $\mathcal{M}$. Setting $z=1$, it follows that $\delta_{i}: x \mapsto d_{i}(x)-\lambda x$ is a superderivation of degree $i$. Namely,

$$
\begin{aligned}
\delta_{i}(x y) & =d_{i}(x y)-\lambda x y \\
& =d_{i}(x) y+(-1)^{i|x|} x d_{i}(y)-2 \lambda x y \\
& =\delta_{i}(x) y+\lambda x y+(-1)^{i|x|} x \delta_{i}(y)-\lambda x y \\
& =\delta_{i}(x) y+(-1)^{i|x|} x \delta_{i}(y)
\end{aligned}
$$

for all $x, y \in \mathcal{H}(\mathcal{A})$. Thus we have the next corollary.

Corollary 3.2. If $\mathcal{R}=\mathcal{A}$, then $d_{i}: \mathcal{A} \rightarrow \mathcal{M}, i=0,1$, satisfies (17). Moreover, if $\mathcal{A}$ and $\mathcal{M}$ are unital, then $\lambda=d_{i}(1) \in \mathcal{Z}(\mathcal{M})$ and there exists a superderivation of degree $i \delta_{i}: \mathcal{A} \rightarrow \mathcal{M}$ such that $d_{i}(x)=\delta_{i}(x)+\lambda x$ for all $x \in \mathcal{H}(\mathcal{A})$.

Corollary 3.3. Let $\mathcal{A}$ be a prime superalgebra containing a nontrivial idempotent in $\mathcal{A}_{0}$ and let $d_{0}: \mathcal{A} \rightarrow \mathcal{A}$ be a $\Phi$-linear map satisfying $d_{0}\left(\mathcal{A}_{0}\right) \subseteq \mathcal{A}_{0}$, $d_{0}\left(\mathcal{A}_{1}\right) \subseteq \mathcal{A}_{1}$, and $\left(d_{0} 2\right)$. Then there exist $\lambda$ in the extended centroid of $\mathcal{A}$ and a superderivation of degree $0 \delta_{0}$ from $\mathcal{A}$ into the central closure of $\mathcal{A}$ such that $d_{0}(x)=\delta_{0}(x)+\lambda x$ for all $x \in \mathcal{A}$. 
Proof. According to our assumption (13) holds true and $\mathcal{A}^{2} \mathcal{I} \neq 0$. Namely, if $\mathcal{A}^{2} \mathcal{I}=0$ then $\mathcal{I}=0$ by the primeness of $\mathcal{A}$. Since $\mathcal{A}$ does not contain a nontrivial central idempotent in $\mathcal{A}_{0}$ it follows that $\mathcal{I} \neq 0$. Let us fix $w \in \mathcal{A}$ and $t \in \mathcal{A}^{2} \mathcal{I}$ such that $w t \neq 0$. By (13) we have

$$
w t z\left(d_{0}(w t)-d_{0}(w) t-w d_{0}(t)\right)=\left(d_{0}(w t)-d_{0}(w) t-w d_{0}(t)\right) z w t
$$

for all $z \in \mathcal{A}$. Using the above equality and [5, Lemma 3.2] there exists $\mu$ in the extended centroid $C$ of $\mathcal{A}$ (for more details on the extended centroid of prime superalgebras see [5]) such that

$$
\mu w t=d_{0}(w t)-d_{0}(w) t-w d_{0}(t) .
$$

But then (13) implies

$$
w t z\left(d_{0}(x y)-d_{0}(x) y-x d_{0}(y)-\mu x y\right)=0
$$

for all $x, y, z \in \mathcal{A}$. By the primeness of $\mathcal{A}$ we obtain

$$
d_{0}(x y)-d_{0}(x) y-x d_{0}(y)=\mu x y
$$

for all $x, y \in \mathcal{A}$. Thereby $d_{0}(x)=\delta_{0}(x)-\mu x$ for all $x \in \mathcal{A}$, where $\delta_{0}$ is a superderivation of degree 0 from $\mathcal{A}$ into the central closure of $\mathcal{A}$. Setting $\lambda=-\mu$ we get the desired conclusion.

In the case when $\mathcal{A}$ is unital $\delta_{0}$ maps $\mathcal{A}$ into itself and $\lambda=d_{0}(1) \in \mathcal{Z}(\mathcal{A})$, where $\mathcal{Z}(\mathcal{A})$ denotes the center of $\mathcal{A}$.

Corollary 3.4. Let $\mathcal{A}$ be a prime superalgebra containing a nontrivial idempotent in $\mathcal{A}_{0}$ and let $d_{1}: \mathcal{A} \rightarrow \mathcal{A}$ be a $\Phi$-linear map satisfying $d_{1}\left(\mathcal{A}_{0}\right) \subseteq \mathcal{A}_{1}$, $d_{1}\left(\mathcal{A}_{1}\right) \subseteq \mathcal{A}_{0}$, and $\left(d_{1} 2\right)$. Then $d_{1}$ is a superderivation of degree 1 .

Proof. According to our assumptions there exist elements $w \in \mathcal{A}$ and $t \in \mathcal{A}^{2} \mathcal{I}$ such that $w t \neq 0$ (see above).

First assume that the odd part $\mathcal{C}_{1}$ of the extended centroid $\mathcal{C}=\mathcal{C}_{0} \oplus \mathcal{C}_{1}$ of $\mathcal{A}$ is zero. Using (13) and [5, Theorem 3.5 (i)] we obtain

$$
w t z\left(d_{1}(x y)-d_{1}(x) y-(-1)^{|x|} x d_{1}(y)\right)=0
$$

for all $x, y, z \in \mathcal{H}(\mathcal{A})$. By the primeness of $\mathcal{A}$ it follows that

$$
d_{1}(x y)-d_{1}(x) y-(-1)^{|x|} x d_{1}(y)=0
$$

for all $x, y \in \mathcal{H}(\mathcal{A})$. Thus we proved that $d_{1}$ is a superderivation of degree 1 on $\mathcal{A}$. 
Now suppose that $\mathcal{C}_{1} \neq 0$. By (14) we have

$$
\begin{aligned}
& (-1)^{|w t|} w t z\left(d_{1}(x y)-d_{1}(x) y-(-1)^{|x|} x d_{1}(y)\right)= \\
& \left(d_{1}(w t)-d_{1}(w) t-(-1)^{|w|} w d_{1}(t)\right) z x y
\end{aligned}
$$

for all $x, y \in \mathcal{H}(\mathcal{A})$ and $z \in \mathcal{A}_{0}$. Using [5, Theorem 3.5 (ii)] the above equality holds true also for all $z \in \mathcal{A}_{1}$. On the other hand we have (by (13))

$$
\begin{aligned}
& -(-1)^{|w t|} w t z\left(d_{1}(x y)-d_{1}(x) y-(-1)^{|x|} x d_{1}(y)\right)= \\
& \left(d_{1}(w t)-d_{1}(w) t-(-1)^{|w|} w d_{1}(t)\right) z x y
\end{aligned}
$$

for all $x, y \in \mathcal{H}(\mathcal{A})$ and $z \in \mathcal{A}_{1}$. Comparing the last two identities we get $w t z\left(d_{1}(x y)-d_{1}(x) y-(-1)^{|x|} x d_{1}(y)\right)=0$ for all $x, y \in \mathcal{H}(\mathcal{A})$ and $z \in \mathcal{A}_{1}$. Analogously we can prove that this identity holds true for all $x, y \in \mathcal{H}(\mathcal{A})$ and $z \in \mathcal{A}_{0}$, which in turn implies that

$$
w t z\left(d_{1}(x y)-d_{1}(x) y-(-1)^{|x|} x d_{1}(y)\right)=0
$$

for all $x, y \in \mathcal{H}(\mathcal{A})$ and $z \in \mathcal{A}$. The primeness of $\mathcal{A}$ implies that

$$
d_{1}(x y)-d_{1}(x) y-(-1)^{|x|} x d_{1}(y)=0
$$

for all $x, y \in \mathcal{H}(\mathcal{A})$. Thereby $d_{1}$ is a superderivation of degree 1 on $\mathcal{A}$.

\section{ACKNOWLEDGMENT}

The authors are grateful to Professor Matej Brešar for sending them the unpublished version of [2].

\section{REFERENCES}

1. M. Brešar, Characterizations of derivations on some normed algebras with involution, J. Algebra, 152 (1992), 454-462.

2. M. Brešar, Characterizing homomorphisms, derivations, and multipliers in rings with idempotents, preprint.

3. M. Brešar, P. Šemrl, Mappings which preserve idempotents, local automorphisms, and local derivations, Canad. J. Math., 45 (1993), 483-496.

4. R. L. Crist, Local derivations on operator algebras, J. Funct. Anal., 135 (1996), 7692.

5. M. Fošner, On the extended centroid of prime associative superalgebras with applications to superderivations, Comm. Algebra, 32 (2004), 689-705. 
6. D. Hadwin, J. Li, Local derivations and local automorphisms, J. Math. Anal. Appl., 290 (2004), 702-714.

7. D. G. Han, S. Y. Wei, Local derivations of nest algebras, Proc. Amer. Math. Soc., 123 (1995), 3095-3100.

8. B. E. Johnson, Local derivations on $C^{*}$-algebras are derivations, Trans. Amer. Math. Soc., 353 (2001), 313-325.

9. R. V. Kadison, Local derivations, J. Algebra, 130 (1990), 494-509.

10. D. Larson, A. R. Sourour, Local derivations and local automorphisms of $\mathcal{B}(X)$, Proc. Symp. Pure Math., 51 (1990), 187-194.

11. L. Molnár, Locally inner derivations of standard operator algebras, Math. Bohem., 121 (1996), 1-7.

12. A. Nowicki, I. Nowosad, Local derivations for quotient and factor algebras of polynomials, Colloq. Math., 97 (2003), 107-116.

13. E. Scholz, W. Timmermann, Local derivations, automorphisms and commutativity preserving maps on $\mathcal{L}^{+}(\mathcal{D})$, Publ. Res. Inst. Math. Sci., 29 (1993), 977-995.

14. J. Schweizer, An analogue of Peetre's theorem in non-commutative topology, Quart. J. Math., 52 (2001), 499-506.

15. V. S. Shulman, Operators preserving ideals in $C^{*}$-algebras, Studia. Math., 109 (1994), 67-72.

16. P. Šemrl, Local automorphisms and derivations on $\mathcal{B}(H)$, Proc. Amer. Math. Soc., 125 (1997), 2677-2680.

17. M. Wiehl, Local derivations on Weyl algebras with one pair of generators, Acta Math. Hungar, 92 (2001), 51-59.

18. J. Zhu, Local derivation of nest algebras, Proc. Amer. Math. Soc., 123 (1995), 739742.

Ajda Fošner

Department of Mathematics and Computer Science,

PeF, University of Maribor,

Koroška cesta 160 ,

SI-2000 Maribor,

Slovenia

E-mail: ajda.fosner@uni-mb.si

\author{
Maja Fošner \\ Institute of Mathematics, Physics and Mechanics, \\ University of Novibor, \\ Jadranska 19, \\ 1000 Ljubljana, \\ Slovenia \\ E-mail: maja.fosner@uni-mb.si
}

